\title{
Invasive Ductal Carcinoma of the Axillary Tail: Report of three Cases and Review of the Literature
}

\author{
Maha Abdel Hadi*, Eiman Shammari, Rahma Shahbahai, Sarah Al Sinan, Khadija Alkhwaja, Roa Al Gowejez \\ and Arwa Al Nabhan
}

Breast surgery, Breast imaging and Oncology divisions, Imam Abdulrahman Bin Faisal University, Saudi Arabia

*Corresponding author: Maha Abdel Hadi, Professor/ Consultant General \& Breast surgeon King Fahd hospital of the University, Saudi Arabia.
Received Date: June 12, 2019

Published Date: August 06, 2019

\section{Abstract}

The aim is to shed light on a rare form of breast cancer occurring at the axillary tail of spence. Presentations may be delayed as many differential diagnoses may be considered. Diagnostic imaging will include the mammography, ultrasound, MRI and tissue diagnosis. We report three cases with similar clinical and histological presentations despite the age difference. Standard radiological and histopathological evaluations were employed to reach the diagnosis of invasive ductal carcinoma (IDC) of the axillary tail.

Keywords: Axillary tail; Invasive ductal carcinoma; Breast cancer

\section{Introduction}

Axillary Breast cancer is a rare entity accounting for 0.1$2 \%$ of all breast cancer cases. It is typically composed of poorly differentiated IDC with occasionally areas of squamous or mesenchymal carcinoma [1]. Axillary malignancy is challenging as there are many differential diagnoses that may require careful evaluation process. The reported differential diagnoses are occult breast carcinoma, accessory breast cancer, axillary tail breast cancer, lymphoma or non-breast metastatic lymphadenopathy [2].

\section{Case 1}

A 28 years old female presented with enlarging left axillary swelling noticed 3months post-partum of her first pregnancy. She was reassured for 9 months attributing it to lactational changes. There was associated progressive pain and limitation of movement of the left shoulder. Menarche at age 14 with regular menstruation and pregnancy with no associated co-morbidities. General examination was unremarkable. Local examination revealed bilateral nodular breasts with no palpable masses. There was a palpable left axillary firm, limited mobility $2 \times 3 \mathrm{~cm}$ mass with no associated tenderness or skin changes.

Ultrasound and mammogram revealed normal bilateral breasts with an axillary tail mass extending to the left axilla with multiple matted axillary nodes on imaging. CT scan confirmed the findings.
Core biopsy of the lesion revealed IDC Patient underwent Excision of Axillary tail with axillary node dissection sparing the breast followed by adjuvant chemo-radiotherapy regimens.

\section{Case 2}

A 32 years old female presented with enlarging right lateral painless breast swelling of 4 months duration. Otherwise she was completely asymptomatic. Menarche at age 12 years with regular menstruation and normal single pregnancy with no associated co-morbidities. General examination was unremarkable. Local examination revealed bilateral nodular breasts with no palpable masses. There was a palpable right non tender axillary tail firm, fixed $3 \times 3 \mathrm{~cm}$ mass with no associated skin changes. Palpable axillary mobile nodes in the ipsilateral axilla. Ultrasound and mammogram revealed a well circumscribed axillary tail mass extending to axillae with multiple nodes on. CT scan confirmed the findings. Core biopsy of the lesion was reported IDC. Patient underwent Excision of Axillary tail with axillary node dissection sparing the breast followed by adjuvant chemo-radiotherapy regimens.

\section{Case 3}

A 69 years old single female, a Known case of schizophrenia and bronchial asthma and both controlled on medication. She Presented with a large left axillary mass of one-year duration with progressive 
increase in size. There was associated hyperpigmentation of the overlying skin otherwise no associated pain or discharge. Menopause at age of 50 years with no history of hormonal replacement therapy. General examination: obese, anxious female otherwise the general examination was unremarkable Local examination: revealed bilateral symmetrical large breasts. The right breast was unremarkable. Left breast: Normal anterior aspect and nipple. There appeared a large breast tail $6 \times 7 \mathrm{~cm}$ firm, nontender fixed mass, associated with thick purple colored skin with peau d' orange (Figure 1).

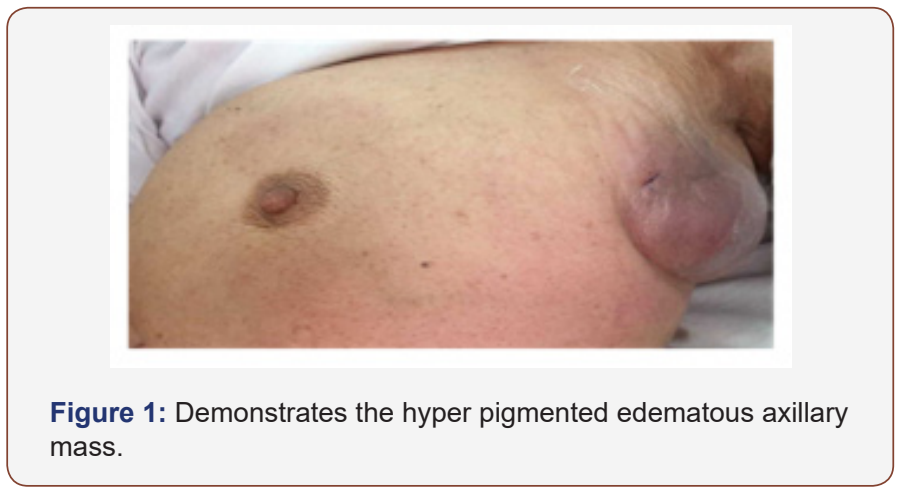

Ultrasound showed a large heterogeneously hypoechoic highly vascular left axillary mass measuring approximately about $10 \times 5.0$ $\mathrm{cm}$ with cystic changes and thickened skin. Highly suggestive of malignancy (Figures 2).

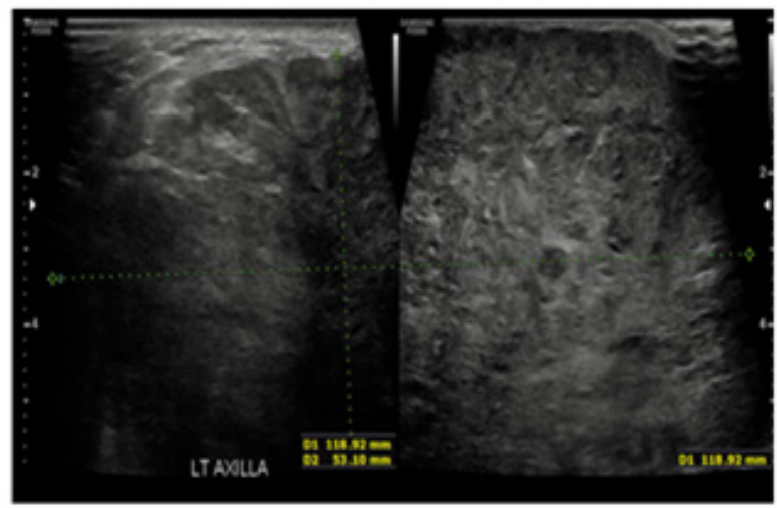

Figure 2: Ultrasound showed a specious large heterogeneously hypoechoic highly axillary mass with cystic changes and thickened skin.

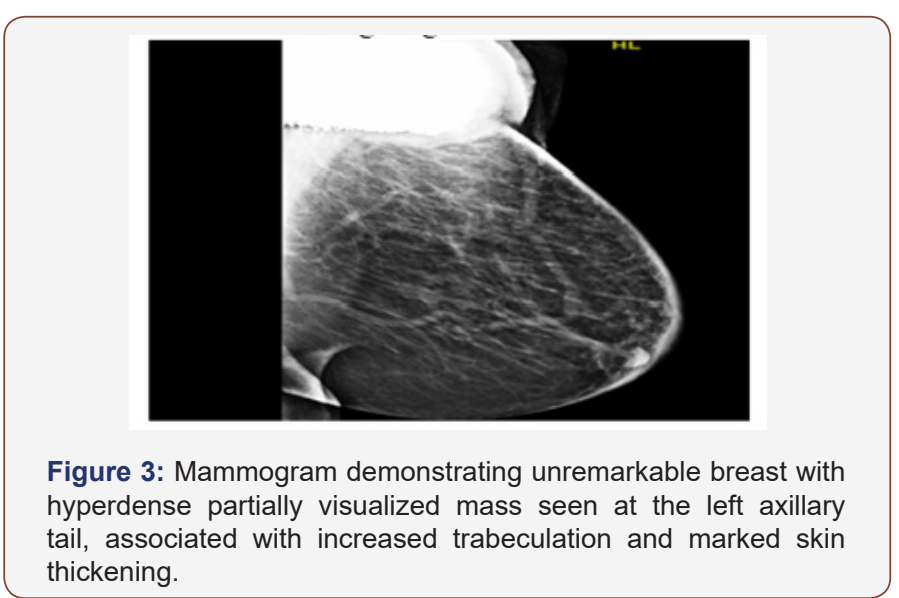

Mammogram was a limited study due to technical reasons demonstrating part of the axillary mass with fat stranding and skin thickening (Figure 3).

Contrast enhanced CT scan both axial and sagittal views of the chest, abdomen and pelvis showing left axillary tail/axilla demonstrated a large irregular heterogeneously enhancing mass with central necrosis measuring $10 \times 7.7 \times 10 \mathrm{~cm}$ at anteroposterior, transverse and craniocaudal diameters respectively attached to the skin with skin invasion. The mass appeared separable from the breast tissue with fat stranding and subcutaneous edema. Multiple Matted ipsilateral axillary lymph nodes with central necrosis were also noted.

\section{Discussion}

Due to the rare occurrence of the carcinoma of the axillary tail it remains an under-recognized entity [1,2]. It possesses a typical clinical, pathological and prognostic feature, promoting the likelihood of nodal metastasis which impacts negatively on both disease progression and survival [3]. Patients usually discover the mass on self-examination and seek medical advice. The standard investigations with ultrasound and mammography are useful modalities yet to anatomical nature of the axillae some limitations are encountered. More sophisticated modalities such as MRI and PET-CT provide accurate delineation facilitating the diagnosis especially in challenging cases $[4,5]$.

IDC is the commonest histological diagnosis especially in malignancies arising from the ectopic breast tissue. Occasionally the diagnosis maybe challenging as other diagnoses such as adnexal skin carcinomas may be difficult to differentiate [6]. The treatment is straight forward with no dispute regarding the axillary dissection. However, mastectomy versus wide local Excision (WLE) remains. Even though recommendations of mastectomy remain, the majority feel that WLE may be adequate $[7,8]$.

\section{Conclusion}

Axillary tail breast cancer is rare and challenging entity that may need special attention in clinical, imaging and pathological practice.

\section{Acknowledgement}

None.

\section{Conflict of Interest}

No conflict of interest.

\section{References}

1. Ampil F, Caldito G, Henderson B, Li B, Kim RH, et al. (2012) Carcinoma of the axillary tail of spence: a case series. Anticancer Res 32(9): 40574059 .

2. Zin W Myint, Sandeep Raparla, Lois K Kamugisha (2015) Metaplastic breast cancer with chondroid differentiation. J Community Hosp Intern Med Perspect 5(4): 28935.

3. Gou ZC, Liu XY, Xiao Y, Zhao S, Jiang YZ, et al. (2018) Decreased survival in patients with carcinoma of axillary tail. Cancer Management and Research 10: 1133-1141. 
4. Okubo M, Tada K, Niwa T (2013) A case of breast cancer in the axillary tail of Spence-enhanced magnetic resonance imaging and positron emission tomography for diagnostic differentiation and preoperative treatment decision. World J Surg Oncol 11: 217.

5. Capobianco G, Spaliviero B, Dessole S, Rocca PC, Cherchi PL, et al. (2007) Lymph node axillary metastasis from occult contralateral infiltrating lobular carcinoma arising in accessory breast: MRI diagnosis. Breast J 13(3): 305-307.
6. Amsler E, Sigal Zafrani B, Marinho E, Aractingi S (2002) Ectopic breast cancer of the axilla. Ann Dermatol Venereol 129(12): 1389-1391.

7. Tajalma WA, Senten LL (2006) The management of ectopic breast cancer: case report. Eur J Gyneaecol Oncol 27 (4): 414-426.

8. Marshall MB, Moynihan JJ, Frost A, Evans SR (1994) Ectopic breast cancer: a case report and literature review. Surg Oncol 3(5): 295-304. 